\title{
Efficacy and Safety of an mHealth App and Wearable Device in Physical Performance for Patients With Hepatocellular Carcinoma: Development and Usability Study
}

Yoon Kim ${ }^{1}, \mathrm{MD}$, MMSc; Jinserk $\mathrm{Seo}^{2}$, BPTh; So-Yeon $\mathrm{An}^{3}$, MPT; Dong Hyun Sinn ${ }^{4}$, MD, PhD; Ji Hye Hwang ${ }^{1}$, $\mathrm{MD}, \mathrm{PhD}$

\footnotetext{
${ }^{1}$ Department of Physical and Rehabilitation Medicine, Samsung Medical Center, Sungkyunkwan University School of Medicine, Seoul, Republic of Korea

${ }^{2}$ Department of Rehabilitation Medicine, Incheon St. Mary's Hospital, The Catholic University of Korea, Incheon, Republic of Korea

${ }^{3}$ Department of Health Science, Korea University Graduate School, Korea University, Seoul, Republic of Korea

${ }^{4}$ Department of Internal Medicine, Samsung Medical Center, Sungkyunkwan University School of Medicine, Seoul, Republic of Korea
}

\section{Corresponding Author:}

Ji Hye Hwang, MD, PhD

Department of Physical and Rehabilitation Medicine

Samsung Medical Center

Sungkyunkwan University School of Medicine

Irwon-Ro 81

Gangnam-Gu

Seoul, 06351

Republic of Korea

Phone: 82234102818

Email: jhlee.hwang@samsung.com

\section{Abstract}

Background: Exercise is predicted to have a positive effect among hepatocellular carcinoma (HCC) patients. However, these patients are hesitant to start and build up an exercise program for one major reason: the vague fear of developing hepatic decompensation, a potentially fatal condition that can lead to death. Integrating mobile health (mHealth) with individualized exercise programs could be a possible option for promoting physical capacity among HCC patients.

Objective: The aim of this study was to evaluate the efficacy and safety of rehabilitation exercises, which have been individually prescribed via an mHealth app, on physical fitness, body composition, biochemical profile, and quality of life among HCC patients.

Methods: A total of $37 \mathrm{HCC}$ patients were enrolled in a 12-week course with an mHealth app program targeted to HCC patients. The wearable wristband device Neofit (Partron Co) was provided to participants, and recorded daily physical data, such as the number of steps, calorie expenditure, exercise time, and heart rate. Each participant was given an individualized rehabilitation exercise program that was prescribed and adjusted at the 6-week midintervention period based on the assessment results. At baseline, 6-week, and 12-week sessions, participants' physical fitness levels (ie, 6-minute walk test, grip strength test, and 30-second chair stand test) were measured. Physical activity levels, as measured by the International Physical Activity Questionnaire-Short Form (IPAQ-SF); body composition (ie, body mass index, body fat percentage, and muscle mass); biochemical profiles; and quality of life, as measured by the European Organization for Research and Treatment of Cancer Quality-of-Life Questionnaire C30, were assessed at baseline and at the end point. At the 6-week midpoint, exercise intensity was individually adjusted.

Results: Of the 37 patients, 31 (84\%) completed the 12-week intervention. Grip strength improved significantly after 12 weeks of the intervention. The 30-second chair stand test and the 6-minute walk test showed significant improvement from 0 to 6 weeks, from 0 to 12 weeks, and from 6 to 12 weeks. Muscle mass and the IPAQ-SF score increased significantly after 12 weeks of the intervention without biochemical deterioration.

Conclusions: Following 12 weeks of mHealth care, including an individually prescribed rehabilitation exercise program, we saw significant improvements in physical fitness, body composition, and physical activity without any complication or biochemical deterioration among compensated HCC patients who had completed therapy. 
(JMIR Mhealth Uhealth 2020;8(3):e14435) doi: 10.2196/14435

\section{KEYWORDS}

mHealth; hepatocellular carcinoma; rehabilitation; exercise; physical fitness; physical activity

\section{Introduction}

Physical activity has been proven to have a positive influence, both biologically and functionally, in patients with cancer, such as breast or prostate cancer [1-3]. Recent studies indicate that even patients undergoing acute cancer treatments can benefit from individualized exercise programs [4]. However, little is known about the role of exercise on hepatocellular carcinoma (HCC), which is the fifth- and seventh-most common cancer worldwide in men and women, respectively [5].

Among the few studies regarding the effect of exercise in HCC, one study reported that continuous regular exercise improved physical ability without deteriorating liver function in HCC patients with chronic liver disease (CLD) [6]. An experimental study using a rat model found that regular physical activity reduced the risk of the primary development of HCC [7]. Although the positive effect of exercise is quite predictable in HCC patients, these patients are hesitant to start and build up an exercise program for one major reason: the vague fear of developing hepatic decompensation. Hepatic decompensation is a potentially fatal condition that can be encountered by patients with HCC, which includes hepatic encephalopathy, esophageal varices, or ascites. Therefore, in order to safely boost exercise capacity, a delicate supporting system is needed to monitor physical activity and alert such vulnerable patients before hepatic decompensation occurs.

Integrating mobile health (mHealth) with individualized exercise programs could be a possible option for promoting physical capacity of HCC patients. Following the explosive increase in smartphone penetration globally, mHealth has been spotlighted as a novel technology for promoting exercise programs, not only among healthy populations but also among patients with various diseases, such as diabetes, heart disease, and cancer [8-10]. Several recent studies have reported the effectiveness of mHealth in patients with solid cancers and survivors of breast cancer and colorectal cancers $[4,11]$. mHealth promotes physical activity among cancer patients by motivating them to exercise and by providing real-time feedback $[12,13]$. Because mHealth can provide large amounts of information and even lower the barrier of communication with health care providers, it is an ideal tool for helping HCC patients to exercise safely outside the hospital.

In this study, we evaluated the efficacy and safety of exercises that were individually prescribed to compensated HCC patients after anticancer therapy. We analyzed changes in physical fitness, body composition, biochemical profile, and quality of life (QoL) after 12 weeks of an mHealth exercise intervention.

\section{Methods}

\section{Participants}

HCC patients who visited the outpatient cancer rehabilitation clinic of a tertiary hospital from November 2017 to February 2018 were prospectively enrolled in this study. The inclusion criteria were as follows: HCC patients aged 19-69 years, patients at stage I or II of the modified Union for International Cancer Control (mUICC) staging system, patients with a Child-Pugh class A or B score, patients who could walk independently for 30 minutes, and patients who had a mobile phone. Exclusion criteria were as follows: patients who required exercise restrictions for severe cardiopulmonary or renal disease, patients with musculoskeletal or neurological deficits, patients with cognitive impairments that interfered with mobile phone utilization, or patients who were unable to give written consent.

\section{App Development}

Based on our previous experience of, and knowledge about, developing mHealth apps for specific cancer patients, we recruited app engineers and five health care professionals from a comprehensive cancer center [4]. Health care professionals were recruited to help in the development of a comprehensive mHealth care system tailored to HCC patients, in order to provide health information, self-monitoring, and connections with health care professionals. The app included the following features: exercise management, nutritional information, health information, my activity analysis, and in-app chat service (see Figure 1).

The exercise management feature provided daily, personalized aerobic and anaerobic exercises based on clinical evidence. The health information content changed daily, including general health information about HCC, medication, adverse effects of anticancer therapy, and nutrition. Real-time communication with a medical professional was available through the in-app chat service. The wearable Internet of Things (IoT) device, which connected with the mobile app, gathered real-time physical data to monitor both physical activity and the health of the participants. A clinical evidence-based care system with the above functions was implemented through the mHealth app. 
Figure 1. App screenshots and configuration. Screenshots of the main page (far left) and exercise management page (middle right) are shown, along with respective diagrams of explanations to the right of each screenshot.

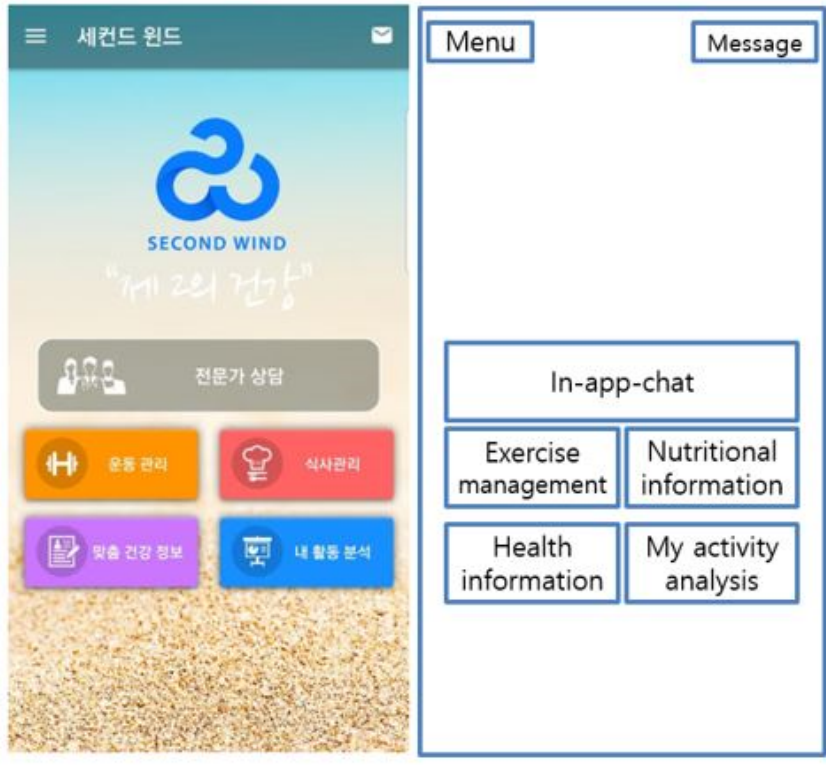

\section{Intervention}

\section{mHealth Care App}

All participants received a 12 -week course of an individually prescribed exercise program through the mHealth app using their own mobile phones and an interconnected wearable IoT device worn on their wrists. The participants were told to download the mobile app to their mobile phones. The mobile app consisted of general medical information on HCC, disease-specific exercise care, nutritional information, and a real-time chat service that directly connected participants to the study coordinator. The wearable IoT device-Neofit (Partron Co) - which we provided to the participants, recorded daily physical data, such as the number of steps, calorie expenditure, exercise time, and heart rate. Each participant was given an individualized rehabilitation exercise program that was prescribed and adjusted at the 6-week midintervention period based on the assessment results.

\section{Individually Prescribed Rehabilitation Exercises}

The patients were recommended to participate in individually tailored regular rehabilitation exercises. Video clips were provided by the app and were composed of warm-up, stretching, aerobic, and muscle-strengthening exercises for the upper and lower extremities. Participants were asked to watch the video clips and perform the exercises daily. During the baseline assessment, participants received instructions for standardized warm-up and stretching exercises and individualized aerobic and muscle-strengthening exercises. The aerobic and muscle-strengthening exercise prescriptions were modified based on each participant's altered physical fitness levels measured at the 6-week midpoint session (see Figure 2).

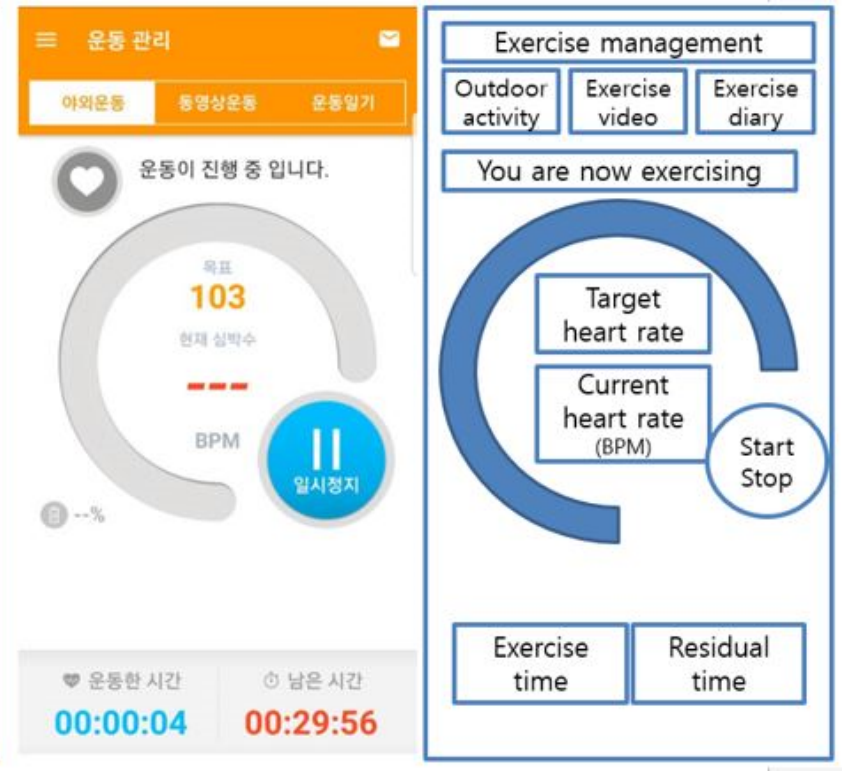

The intensity (ie, light walking, light running, mountain climbing, and cycling) and target heart rate for the aerobic exercise were set from the results of the 6-minute walk test (6MWT). This result was compared with an individualized reference value calculated using each participant's age, height, and weight [14]. If the 6MWT result was higher than the individualized reference value, the recommended exercise intensity and target heart rate were increased.

All the major muscle groups of the upper extremities, lower extremities, and trunk were included in the muscle-strengthening exercise program. Three steps of resistance exercises (ie, maximum, moderate, and minimum resistance) were provided. The results of the 30-second chair stand test and grip strength test were compared with reference values based on the healthy, normal, Korean population from the Korea Sports Promotion Foundation. Appropriate intensity levels for the strengthening exercises were determined according to those results.

After 6 weeks of participation, fitness levels (ie, 6MWT, grip strength test, and 30-second chair stand test) were assessed in all participants. According to the results of this midpoint assessment, the intensity of the aerobic exercise and level of strengthening exercises were adjusted. The target intensity and target heart rate of the aerobic exercise were altered according to the results of the 6MWT. We increased the recommended exercise level if the participant's 6MWT distance increased. The level of the resistance exercises was altered according to the results of the grip strength test and the 30-second chair stand test. Participants' conditions, as a result of their exercise programs, were communicated through the app's real-time chat service. Participant compliance with the exercise program was checked by calculating the total aerobic exercise time completed and the number of resistance exercise video clips watched. 
Figure 2. Procedure for adjusting participants' individually prescribed rehabilitation exercise programs in the mHealth app. 6MWT: 6-minute walk test.

\title{
Baseline
}

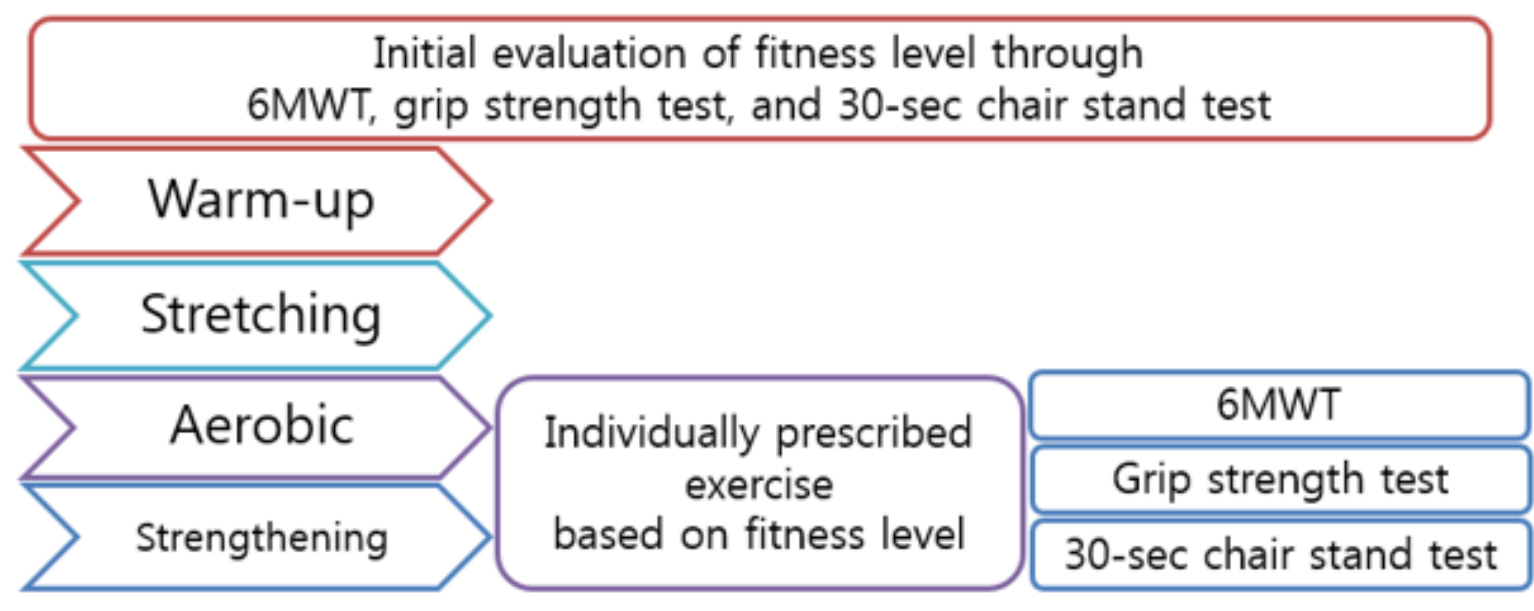

\section{6-week midintervention}

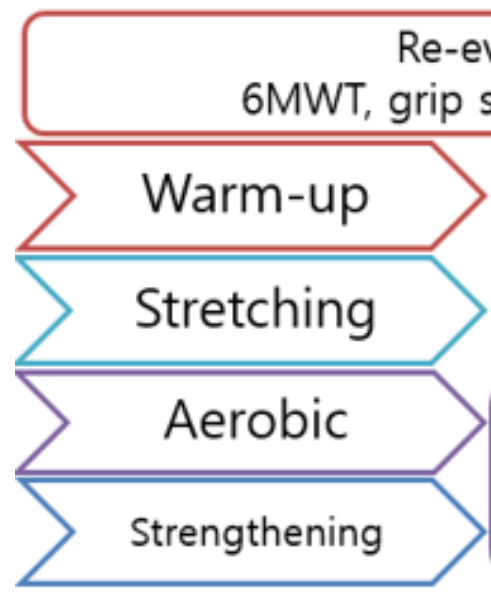

\begin{tabular}{|c|}
\hline 6MWT \\
\hline Grip strength test \\
\hline $30-$ sec chair stand test \\
\hline
\end{tabular}

\section{2-week end of intervention}

\author{
Final evaluation of fitness level through \\ $6 \mathrm{MWT}$, grip strength test, and $30-\mathrm{sec}$ chair stand test
}

\begin{abstract}
Measures
At baseline and at the end point of the study, we collected demographic characteristics and related medical data (ie, blood tests and body composition analyses); in addition, participants completed questionnaires for physical activity and QoL. The outcome measures for physical fitness level were performed at baseline, midintervention (ie, 6 weeks), and at the intervention end point (ie, 12 weeks).

Blood tests, completed at baseline and at the study end point, consisted of a complete blood count (ie, white blood cell count, hemoglobin, and platelets), chemistry panel (ie, albumin,
\end{abstract}

cholesterol, total bilirubin, creatinine, and osteocalcin), liver function tests (ie, aspartate aminotransferase, alanine aminotransferase, alkaline phosphatase, and gamma glutamyl transferase), and a coagulation study (ie, prothrombin time [international normalized ratio]).

Data for the body composition analyses were collected using a bioelectric impedance device—Inbody 720 (Biospace). Muscle mass and body fat percentages were obtained. Body mass index (BMI) was calculated as body weight $/$ height $\left(\mathrm{kg} / \mathrm{m}^{2}\right)$.

The International Physical Activity Questionnaire-Short Form (IPAQ-SF) was used to measure participants' physical activity 
[15]. This questionnaire contains nine questions about the minutes per day or days per week spent doing activities of vigorous and moderate intensity and about time spent walking or sitting during the past 7 days. Using this questionnaire, we calculated the total number of metabolic equivalents (METs) per week. The calculated physical activity was classified into three groups according to the IPAQ-SF scoring system: inactive, minimally active, and highly active. The inactive group consumed less than 600 METs of physical activity, the minimally active group consumed more than 600 METs but less than 3000 METs, and the highly active group consumed a minimum of 3000 METs.

QoL related to general health was assessed by the European Organization for Research and Treatment of Cancer Quality-of-Life Questionnaire C30 (EORTC-QLQ-C30) [16]. This questionnaire contains 30 items regarding general health status, five functional scales (ie, physical, role, cognitive, emotional, and social functioning), three symptom scales (ie, fatigue, pain, and nausea or vomiting), and six single-item scales (ie, dyspnea, appetite loss, constipation, diarrhea, financial difficulties, and insomnia). Each scale includes a different set of items, which are calculated using specific coding procedures. Higher scores for the general health status and the functional scales imply positive results, whereas the symptom and the single-item scales are interpreted inversely [17].

Physical fitness was measured with the grip strength test, the 30-second chair stand test, and the 6MWT. A hand-held dynamometer-SH 5001 (Saehan Corp)—was used to assess upper-extremity muscle strength. In an upright posture with slightly abducted arm and slightly flexed elbow, the participants were instructed to hold the dynamometer with the arm and wrist both in a neutral position. After holding it for 3 seconds, maximal power was measured. This grip strength test was repeated three times and the average power was recorded [18]. Lower-limb strength was assessed by the 30 -second chair stand test, which counts the maximum number of times a participant can stand up from a chair in 30 seconds. At the start, each participant was seated straight up in a chair without leaning on the backrest and with both arms folded across the chest. For 30 seconds, they repeated complete stand-up and sit-down motions as quickly as possible, and the total number of complete standing motions was counted [19]. To measure the level of cardiopulmonary endurance, the 6MWT was conducted in a 15.2-meter hallway. The total distance walked at maximal speed for 6 minutes was recorded. The physical fitness data were compared with age-specific, normal, Korean fitness values from the National Fitness 100 project from the Korean government [20].

\section{Statistical Analysis}

SPSS Statistics for Windows, version 24.0 (IBM Corp), was used for statistical analyses, and statistical significance was established as $P<.05$. General and clinical subject characteristics were analyzed using descriptive statistics. One-way, repeated-measures analysis of variance (ANOVA) was used to analyze changes in physical fitness over time. Because body composition, physical activity levels, and biochemical profiles were checked at baseline and after 12 weeks of the intervention, the paired $t$ test or the Wilcoxon signed-rank test was used to determine the effects of the exercise intervention.

\section{Ethics Approval and Consent to Participate}

All decisions regarding this study were approved by the Institutional Review Board of Samsung Medical Center after a complete review of clinical trial protocols (approval number: 2017-06-050). All participants provided written informed consent.

\section{Results}

\section{Demographics and Clinical Characteristics}

A total of 37 patients diagnosed with HCC were enrolled in this study. Among the 37 patients, 31 of them-26 (84\%) males and $5(16 \%)$ females-completed the 12-week intervention using the mHealth app program on a mobile phone with an interconnected wearable device. The 6 patients $(6 / 37,16 \%)$ who did not use the mHealth app and did not respond to our communications regarding the 6-week midintervention evaluation were removed from the study. The demographic and clinical characteristics of the study participants are presented in Table 1. Their mean age was 56.7 years (SD 7.7) and 84\% $(26 / 31)$ of them were male. Participants' mean BMI was 25.39 $\mathrm{kg} / \mathrm{m}^{2}$ (SD 3.00) and mean muscle mass was $28.98 \mathrm{~kg}$ (SD 5.20). Participants' initial mean body fat percentage was $26.29 \%$ (SD 8.01 ), which is in the upper-normal range (normal range: $18.0 \%-28.0 \%$ ). As for underlying CLD, 24 out of 31 (77\%) patients had liver cirrhosis and $7(23 \%)$ had chronic hepatitis. In total, $74 \%$ (23/31) of our patients had been diagnosed with HCC for more than 1 year, and 4 patients out of 31 (13\%) were long-term patients of more than 5 years. In total, $19 \%$ (6/31) of patients had previously experienced hepatic decompensation, mainly variceal hemorrhage. The most common therapeutic method was surgery $(17 / 31,55 \%)$, followed by combination treatments $(11 / 31,35 \%)$ and locoregional therapies (ie, radiofrequency ablation or transarterial chemoembolization) $(3 / 31,10 \%)$. 
Table 1. Demographic and clinical characteristics of participants, $(\mathrm{N}=31)$.

\begin{tabular}{|c|c|}
\hline Characteristic & Value \\
\hline Age (years), mean (SD) & $56.7(7.7)$ \\
\hline \multicolumn{2}{|l|}{ Gender, n (\%) } \\
\hline Male & $26(84)$ \\
\hline Female & $5(16)$ \\
\hline Height (cm), mean (SD) & $166.9(8.2)$ \\
\hline Weight (kg), mean (SD) & $71.0(11.3)$ \\
\hline Body mass index $\left(\mathrm{kg} / \mathrm{m}^{2}\right)$, mean $(\mathrm{SD})$ & $25.39(3.00)$ \\
\hline Body fat (\%), mean (SD) & $26.29(8.01)$ \\
\hline Muscle mass (kg), mean (SD) & $28.98(5.20)$ \\
\hline \multicolumn{2}{|l|}{ Underlying chronic liver disease, n (\%) } \\
\hline Liver cirrhosis & $24(77)$ \\
\hline Chronic viral hepatitis & $5(16)$ \\
\hline Nonalcoholic fatty liver disease & $1(3)$ \\
\hline Alcoholic liver disease & $1(3)$ \\
\hline \multicolumn{2}{|l|}{ Diagnosis date, $\mathrm{n}(\%)$} \\
\hline $0-6$ months ago & $5(16)$ \\
\hline 6 months- 1 year ago & $3(10)$ \\
\hline $1-3$ years ago & $11(35)$ \\
\hline $3-5$ years ago & $8(26)$ \\
\hline More than 5 years ago & $4(13)$ \\
\hline \multicolumn{2}{|l|}{ Comorbidity, n (\%) } \\
\hline Diabetes mellitus & $7(23)$ \\
\hline Hypertension & $10(32)$ \\
\hline Dyslipidemia & $1(3)$ \\
\hline Cardiopulmonary disease & $1(3)$ \\
\hline \multicolumn{2}{|l|}{ Previous experience of hepatic decompensation, $n(\%)$} \\
\hline None & $25(81)$ \\
\hline More than once & $6(19)$ \\
\hline \multicolumn{2}{|l|}{ Treatment, $\mathrm{n}(\%)$} \\
\hline Locoregional therapies (ie, transarterial chemoembolization or radiofrequency ablation) & $3(10)$ \\
\hline Surgery & $17(55)$ \\
\hline Combination treatment & $11(35)$ \\
\hline
\end{tabular}

\section{Physical Fitness Measures and Compliance Rate}

Table 2 and Figure 3 present the serial changes in the objective physical fitness measures at baseline and after using the mHealth app with individually prescribed rehabilitation exercises for 6 and 12 weeks. From baseline to final measurement, grip strength was graded as poor according to the Korean national fitness normal value for 55-59-year-old males. However, compared with baseline, grip strength did improve significantly after 12 weeks of the intervention $(P=.02)$. The 30 -second chair stand test also showed significant improvement after 6 and 12 weeks, compared with baseline $(P<.001$ and $P<.001$, respectively), and from 6 to 12 weeks $(P<.001)$. Likewise, the 6MWT showed significant improvement after 6 and 12 weeks, compared with baseline ( $P<.001$ and $P<.001$, respectively), and from 6 to 12 weeks $(P=.01)$.

For aerobic exercise, the stiffest decline in compliance took place at 6-7 weeks, whereas for the strengthening exercises, the most rapid decline was at $4-5$ weeks $(\delta=9.68 \%$ and $\delta=11.29 \%$, respectively). 
Table 2. Changes in physical fitness measures.

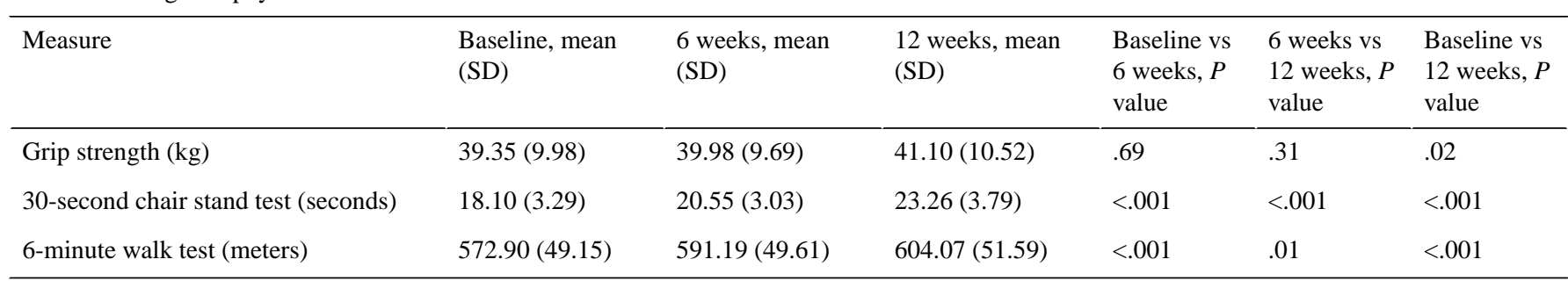

Figure 3. Changes in physical fitness measures. BL: baseline; wks: weeks.

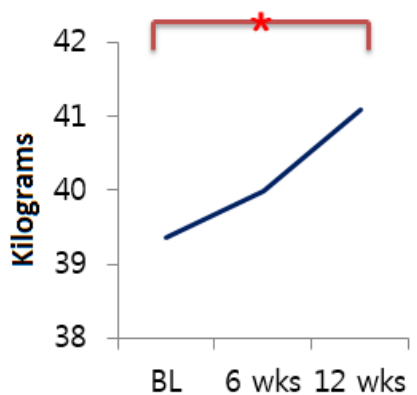

Grip strength (dominant hand)$$
* P<.05
$$

\section{Body Composition and Self-Reported Physical Activity}

As shown in Table 3 and Figure 4, muscle mass increased significantly after 12 weeks of prescribed exercise $(P=.03)$. The change in BMI was insignificant, and body fat percentage declined but without statistical significance $(P=.08$ and $P=.51$, respectively). At baseline, the mean, weekly, physical activity

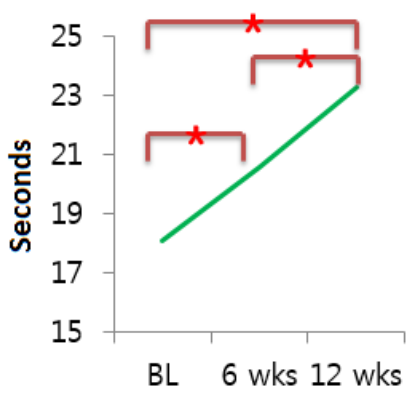

30-second chair stand test

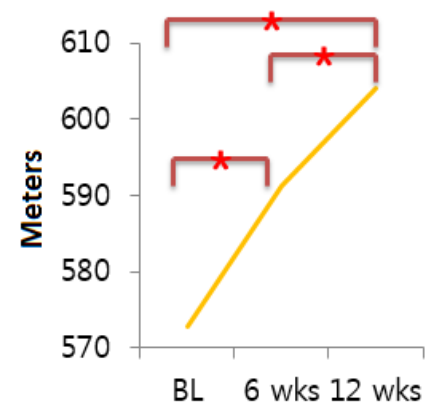

6-minute walk test

Table 3. Changes in body composition and self-reported physical activity.

\begin{tabular}{lll}
\hline Measure & Baseline, mean (SD) & 12 weeks, mean (SD) \\
\hline Body mass index $\left(\mathrm{kg} / \mathrm{m}^{2}\right)$ & $25.39(3.00)$ & $25.57(3.08)$ \\
Body fat $(\%)$ & $26.29(8.01)$ & $26.07(7.86)$ \\
Muscle mass $(\mathrm{kg})$ & $28.98(5.15)$ & $29.34(5.31)$ \\
IPAQ-SF $^{\mathrm{a}}\left(\right.$ METs $\left.^{\mathrm{b}}\right)$ & $2031.95(2236.60)$ & $3479.71(2640.08)$
\end{tabular}

aPAQ-SF: International Physical Activity Questionnaire-Short Form.

${ }^{\mathrm{b}}$ METs: metabolic equivalents.

Figure 4. Changes in body composition and self-reported physical activity. BL: baseline; BMI: body mass index; IPAQ-SF: International Physical Activity Questionnaire-Short Form; METs: metabolic equivalents.

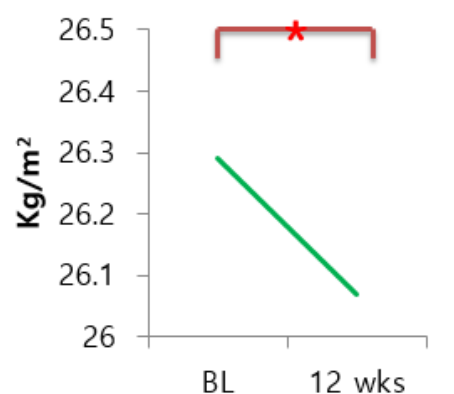

BMI

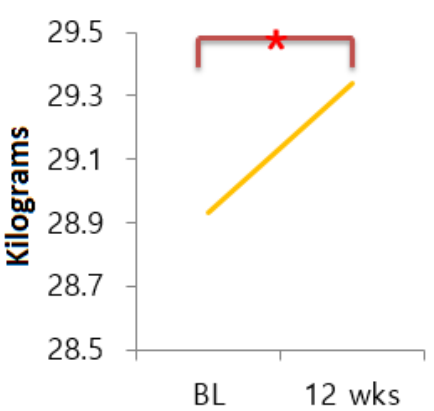

Muscle mass

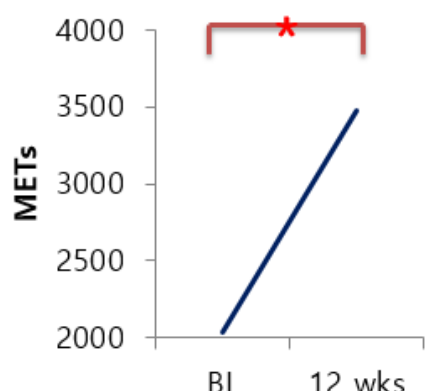

IPAQ-SF

$* P<.05$ 


\section{Biochemical Profile and Complications}

Serum levels of aspartate aminotransferase, alanine aminotransferase, alkaline phosphatase, and gamma glutamyl transferase, which indicate liver function, showed no significant change after 12 weeks of exercise. Insignificant results were seen in albumin and creatinine, which are synthesized in liver cells, and in total bilirubin and prothrombin time (ie, international normalized ratio). No significant difference was observed in osteocalcin, which is inversely related to nonalcoholic fatty liver disease (NAFLD); cholesterol, which increases in NAFLD; or complete blood count. During 12 weeks of exercise, no complications were reported through the in-app chat service, including hepatic decompensation in the form of variceal hemorrhage, ascites, jaundice, or hepatic encephalopathy.

\section{Quality of Life}

Table 4 presents the changes in QoL after 12 weeks of monitored, individualized exercise via our mHealth app. Based on the EORTC-QLQ-C30, the most common symptom was fatigue, followed by dyspnea and insomnia. After 12 weeks of individually prescribed exercise through the mHealth app, all symptoms improved insignificantly, except pain, which decreased significantly $(P=.04)$. Global health status and all functional scales trended toward improvement after 12 weeks of the intervention, though without statistical significance.

Table 4. Changes in quality of life.

\begin{tabular}{|c|c|c|c|}
\hline EORTC-QLQ-C30 item & Score at baseline, mean (SD) & Score at 12 weeks, mean (SD) & $P$ value \\
\hline Global health status and quality of life $\mathrm{e}^{\mathrm{b}}$ & $72.50(17.52)$ & $74.44(17.63)$ & .43 \\
\hline \multicolumn{4}{|l|}{ Functional scale $^{b}$} \\
\hline Physical functioning & $85.77(10.31)$ & $87.55(11.30)$ & .43 \\
\hline Role functioning & $87.22(13.62)$ & $89.44(16.65)$ & .38 \\
\hline Emotional functioning & $84.16(18.48)$ & $87.50(12.90)$ & .28 \\
\hline Cognitive functioning & 80.55 (13.19) & $86.11(11.64)$ & .06 \\
\hline Social functioning & $85.55(24.65)$ & $93.33(12.06)$ & .08 \\
\hline \multicolumn{4}{|l|}{ Symptom scale or single item ${ }^{c}$} \\
\hline Fatigue & $25.18(12.69)$ & $23.33(15.25)$ & .48 \\
\hline Nausea and vomiting & $2.22(5.76)$ & $2.17(6.31)$ & .66 \\
\hline Pain & $10.55(14.17)$ & $6.11(11.14)$ & .04 \\
\hline Dyspnea & $22.22(26.74)$ & $17.77(24.34)$ & .35 \\
\hline Insomnia & $15.55(22.71)$ & $14.44(20.86)$ & .80 \\
\hline Appetite loss & $6.66(16.14)$ & $5.55(12.63)$ & .71 \\
\hline Constipation & $12.00(17.83)$ & $11.11(15.98)$ & .80 \\
\hline Diarrhea & $12.00(17.83)$ & $11.11(15.98)$ & .71 \\
\hline Financial difficulties & $13.33(25.67)$ & $7.77(16.80)$ & .20 \\
\hline
\end{tabular}

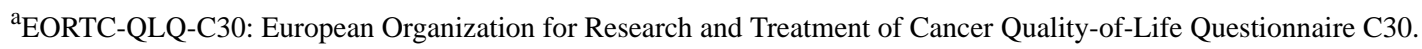

${ }^{\mathrm{b}}$ Higher scores imply positive results.

${ }^{\mathrm{c}}$ Lower scores imply positive results.

\section{Discussion}

\section{Principal Findings}

In this study, we found that it was safe and effective for compensated HCC patients who have completed anticancer therapy to undergo 12 weeks of individually prescribed rehabilitation exercises using our mHealth app and interconnected IoT wearable device. Our surveillance system, composed of the wearable IoT device and real-time communication chat service with health care professionals, found no complications or biochemical deterioration during the 12 weeks of the intervention. Compared to baseline, statistically significant improvements were found in the physical fitness measures (ie, grip strength, 30-second chair stand test, and 6MWT), body composition (ie, muscle mass), self-reported amount of physical activity (ie, IPAQ-SF), and pain. All symptoms trended toward improvement in the QoL scales (ie, EORTC-QLQ C30) after 12 weeks of the intervention. Compliance with the aerobic and strengthening exercises decreased slightly but was maintained overall throughout the intervention period. To the best of our knowledge, this was the first study to use an mHealth app and interconnected IoT device for individually prescribed rehabilitation exercises in compensated HCC patients after anticancer treatment.

Growing interest had led to many studies investigating the association of cancer and exercise. Increased risks for several 
solid cancers, including breast, endometrial, prostate, and colorectal, were inversely associated with physical activity [21]. Recent studies indicate that even after diagnosis with those solid cancers, regular exercise resulted in more survival years and a lower tumor recurrence rate [22-24]. The biochemical mechanisms of such findings are still being discovered, but exercise-dependent regulation of the tumor microenvironment is currently held to be the primary mechanism. In experimental models, chronic exposure to exercise stimulated interorgan signaling through the complex control of hormones, cytokines, and growth factors. Consequently, reprogramming of the systemic milieu was stimulated, resulting in regulation of the tumor microenvironment through angiogenesis, immune regulation, and metabolism [25].

The potential benefit of exercise on liver disease was first studied in NAFLD, of which the key pathophysiological mechanism is insulin resistance [26]. In terms of HCC, a Taiwanese cohort study [27], which was later confirmed by the National Institutes of Health [28], found that a degree of physical activity correlated with a decline in HCC risk. The antitumoral effect of exercise on HCC is mainly associated with decreases in body weight, insulin resistance, and chronic inflammation [29]. In a previous experimental study with a rodent model of nonalcoholic steatohepatitis, regular exercise inhibited HCC development by stimulating adenosine monophosphate-activated protein kinase and inhibiting mammalian target of rapamycin complex 1 [7].

However, only a few studies have considered the potential therapeutic effects of exercise after the development of HCC. One Japanese study had HCC patients work out at their anaerobic thresholds from 1 month preoperation until 6 months postoperation. The exercise group showed decreased whole-body mass, fat mass, and fasting serum insulin; they even showed improvement in the anaerobic threshold, peak oxygen consumption, and insulin resistance [30]. Contrary to our intervention period of 12 weeks, the Japanese study's program began before hepatectomy and lasted for 6 months postoperation. Also, that study's exercise intervention was targeted to the anaerobic threshold and consisted only of stretching and walking, whereas our study's exercise protocol combined stretching, aerobic, and muscle-strengthening exercises. Both studies adjusted the exercise program during the intervention. Most importantly, compared to the Japanese patients who visited the study center three times a week for 60-minute exercise sessions with an exercise trainer, our participants exercised freely outside the hospital via the mHealth program. In our study, exercise compliance and safety were monitored via the mHealth devices.

Another study reported that a median of 13 days of in-hospital exercise among HCC patients with underlying CLD did not worsen the Child-Pugh class, maintained the 6MWT distance, and significantly improved heart rate variability [6]. Compared to the thorough exercise program for upper and lower extremities in our study protocol, the exercise program of that previous study contained stretching, strengthening, and balance training for only the lower extremities. Unlike our study, the exercises in the previous study were not tailored according to individual fitness levels nor adjusted during the intervention period. Also, those exercises were initiated 1 day after anticancer treatment for HCC. Though the previous study evaluated 6MWT and biochemical profiles by blood sampling, the relatively short exercise period (ie, median 7.5 days) of that in-hospital study was not long enough to show physiological or biological changes.

Contrary to malignancies that occur without any underlying condition, HCC commonly arises in cirrhotic liver or viral hepatitis. Most HCC patients suffer from CLD for several years before developing HCC, so their QoL is low and worsens after cancer treatment. One global survey study found that among HCC patients, $81 \%$ receiving sorafenib (ie, tyrosine kinase inhibitor), $45 \%$ receiving selective internal radiation therapy, and $32 \%$ receiving transarterial chemoembolization reported impaired QoL [31]. Our unpublished previous survey study also found that CLD patients, including those with HCC, reported a low level of health-related QoL and physical activity, being minimally active. Boosting the low physical fitness levels of chronically ill HCC patients is a complex process, involving both motivating and safely leading these patients to exercise properly.

As the chronicity of the underlying CLD in HCC patients deteriorates their general physical activity levels, we used an mHealth app via a mobile phone and interconnected wearable IoT device. This system allowed close monitoring of patients' vital signs and exercise compliance, which allowed all participants to complete the 12-week exercise program without any complications or biochemical deterioration. Despite increasing interest and studies on mHealth for cancer patients, studies involving the use of IoT devices among cancer patients are scarce. A few studies using wearable IoT devices and mHealth apps were conducted to promote physical activity in breast cancer survivors and childhood cancer survivors [32-34]. Only two studies used an IoT device among cancer patients under treatment: one is our previous study involving colorectal cancer patients receiving chemotherapy, and the other study estimated the symptom severity of chemotherapy with a wearable IoT device in gastrointestinal cancer patients $[4,35]$. We are the first to apply mHealth with an IoT device to HCC patients. According to the satisfaction survey after 12 weeks of our mHealth program for HCC patients, $84 \%$ of participants reported medium-to-high satisfaction with the mHealth program. A total of $87 \%$ of participants wanted to continue to use the program after the study ended.

This study has several limitations. First, the study population was small and the intervention period was short. In most of the previously conducted feasibility or pilot studies regarding new protocols of exercise interventions among a cancer population, 10-30 patients were enrolled [36-39]. Since this was the first study to apply an IoT-based individualized exercise program among compensated HCC patients who had completed therapy, we considered that the final participant number of 31 was sufficient. The intervention period was selected based on previous interventional studies using mobile apps among cancer patients. Most studies were conducted for 12 weeks or 3 months $[11,18,40]$. Further studies with large sample sizes and longitudinal intervention periods will support the results of this study. Second, the completion rate of this study was low. The 
completion rate in exercise intervention studies of cancer populations differs based on the traits of the population, such as dominant gender, mean age, severity of cancer, general condition, needs of exercise, and type of ongoing anticancer therapy. For example, one study that compared the physical fitness effect of mHealth and conventional exercise using a brochure among breast cancer patients-all female patients, mean age 50.3 years, and 12-week follow-up_-showed a $95.2 \%$ completion rate [18]. In contrast, $73.9 \%$ of participants diagnosed with prostate cancer-all male patients, mean age 68.4 years, and 12-week follow-up-completed the 12-week course of a home-based exergaming intervention [41]. The completion rate of $84 \%(31 / 37)$ in this study $-84 \%$ male patients, mean age 56.7 years, and 12-week follow-up-is not lower than that of the previous prostate cancer exercise intervention. Also, without a previous study of a 12-week exercise intervention among HCC patients, it is difficult to confirm that the completion rate of our study is low. Third, we used no control group in this study. Inclusion of a sex- and age-matched control group could have strengthened our results. However, as this was the first study to apply an mHealth-based exercise program to HCC patients, comparing the outcome measures of the initial, 6-week, and 12-week visits of the
mHealth exercise group without a control group yielded significant results. Finally, because this study was conducted among compensated HCC patients after cancer therapy, it would be inappropriate to generalize these results. Further studies with a larger sample size and a randomized controlled study involving HCC patients with diverse medical statuses are needed.

\section{Conclusions}

In this study, compensated HCC patients after therapy underwent 12 weeks of comprehensive cancer care through an mHealth app. The app provided general medical information on HCC, nutritional information, real-time communication with a health care provider, and an individually prescribed rehabilitation exercise program. The exercise program was monitored through an IoT device. The intervention significantly improved physical fitness, body composition, and self-reported physical activity without any complications or biochemical deterioration. We found it safe and effective for compensated HCC patients after cancer treatment to exercise using the mobile app and interconnected IoT wearable device. This advanced technology allows effective and practical, patient-centered, supervised home exercise therapy to be an alternative to conventional, unfeasible, hospital-oriented exercise programs.

\section{Conflicts of Interest}

None declared.

\section{References}

1. Wirtz P, Baumann FT. Physical activity, exercise and breast cancer: What is the evidence for rehabilitation, aftercare, and survival? A review. Breast Care (Basel) 2018 Apr;13(2):93-101 [FREE Full text] [doi: 10.1159/000488717] [Medline: 29887785]

2. Okumatsu K, Tsujimoto T, Wakaba K, Seki A, Kotake R, Yamauchi T, et al. Effects of a combined exercise plus diet program on cardiorespiratory fitness of breast cancer patients. Breast Cancer 2019 Jan;26(1):65-71. [doi: 10.1007/s12282-018-0889-x] [Medline: 29992486]

3. Campos C, Sotomayor P, Jerez D, González J, Schmidt CB, Schmidt K, et al. Exercise and prostate cancer: From basic science to clinical applications. Prostate 2018 Jun;78(9):639-645. [doi: 10.1002/pros.23502] [Medline: 29569731]

4. Cheong IY, An SY, Cha WC, Rha MY, Kim ST, Chang DK, et al. Efficacy of mobile health care application and wearable device in improvement of physical performance in colorectal cancer patients undergoing chemotherapy. Clin Colorectal Cancer 2018 Jun;17(2):e353-e362. [doi: 10.1016/j.clcc.2018.02.002] [Medline: 29551558]

5. Mittal S, El-Serag HB. Epidemiology of hepatocellular carcinoma: Consider the population. J Clin Gastroenterol 2013 Jul;47 Suppl:S2-S6 [FREE Full text] [doi: 10.1097/MCG.0b013e3182872f29] [Medline: 23632345]

6. Koya S, Kawaguchi T, Hashida R, Goto E, Matsuse H, Saito H, et al. Effects of in-hospital exercise on liver function, physical ability, and muscle mass during treatment of hepatoma in patients with chronic liver disease. Hepatol Res 2017 Mar;47(3):E22-E34. [doi: 10.1111/hepr.12718] [Medline: 27062043]

7. Piguet A, Saran U, Simillion C, Keller I, Terracciano L, Reeves HL, et al. Regular exercise decreases liver tumors development in hepatocyte-specific PTEN-deficient mice independently of steatosis. J Hepatol 2015 Jun;62(6):1296-1303. [doi: 10.1016/j.jhep.2015.01.017] [Medline: 25623824]

8. Whitehead L, Seaton P. The effectiveness of self-management mobile phone and tablet apps in long-term condition management: A systematic review. J Med Internet Res 2016 May 16;18(5):e97 [FREE Full text] [doi: 10.2196/jmir.4883] [Medline: 27185295]

9. Sankaran S, Dendale P, Coninx K. Evaluating the impact of the HeartHab app on motivation, physical activity, quality of life, and risk factors of coronary artery disease patients: Multidisciplinary crossover study. JMIR Mhealth Uhealth 2019 Apr 04;7(4):e10874 [FREE Full text] [doi: 10.2196/10874] [Medline: 30946021]

10. Wu X, Guo X, Zhang Z. The efficacy of mobile phone apps for lifestyle modification in diabetes: Systematic review and meta-analysis. JMIR Mhealth Uhealth 2019 Jan 15;7(1):e12297 [FREE Full text] [doi: 10.2196/12297] [Medline: 30664494]

11. Geng Z, Wu F, Zhang Y, Wang J, Wang J, Liu H, et al. Mobile physical activity intervention for breast cancer patients during chemotherapy. Stud Health Technol Inform 2018;250:236. [Medline: 29857447] 
12. Mercer K, Giangregorio L, Schneider E, Chilana P, Li M, Grindrod K. Acceptance of commercially available wearable activity trackers among adults aged over 50 and with chronic illness: A mixed-methods evaluation. JMIR Mhealth Uhealth 2016 Jan 27;4(1):e7 [FREE Full text] [doi: 10.2196/mhealth.4225] [Medline: 26818775]

13. Lyons EJ, Lewis ZH, Mayrsohn BG, Rowland JL. Behavior change techniques implemented in electronic lifestyle activity monitors: A systematic content analysis. J Med Internet Res 2014 Aug 15;16(8):e192 [FREE Full text] [doi:

10.2196/jmir.3469] [Medline: 25131661]

14. Enright PL, Sherrill DL. Reference equations for the six-minute walk in healthy adults. Am J Respir Crit Care Med 1998 Nov;158(5 Pt 1):1384-1387. [doi: 10.1164/ajrccm.158.5.9710086] [Medline: 9817683]

15. Craig CL, Marshall AL, Sjöström M, Bauman AE, Booth ML, Ainsworth BE, et al. International physical activity questionnaire: 12-country reliability and validity. Med Sci Sports Exerc 2003 Aug;35(8):1381-1395. [doi: 10.1249/01.MSS.0000078924.61453.FB] [Medline: 12900694]

16. Sternby Eilard M, Hagström H, Mortensen KE, Wilsgaard T, Vagnildhaug OM, Dajani O, et al. Quality of life as a prognostic factor for survival in hepatocellular carcinoma. Liver Int 2018 May;38(5):885-894. [doi: 10.1111/liv.13593] [Medline: 28941130]

17. Fayers PA, Bjordal K, Groenvold M, Curran D, Bottomley A, on behalf of the EORTC Quality of Life Group. EORTC QLQ-C30 Scoring Manual. 3rd edition. Brussels, Belgium: European Organisation for Research and Treatment of Cancer (EORTC); 2001. URL: https://www.eortc.org/app/uploads/sites/2/2018/02/SCmanual.pdf [accessed 2020-01-29]

18. Uhm KE, Yoo JS, Chung SH, Lee JD, Lee I, Kim JI, et al. Effects of exercise intervention in breast cancer patients: Is mobile health (mHealth) with pedometer more effective than conventional program using brochure? Breast Cancer Res Treat 2017 Feb;161(3):443-452. [doi: 10.1007/s10549-016-4065-8] [Medline: 27933450]

19. Millor N, Lecumberri P, Gómez M, Martínez-Ramírez A, Izquierdo M. An evaluation of the 30-s chair stand test in older adults: Frailty detection based on kinematic parameters from a single inertial unit. J Neuroeng Rehabil 2013 Aug 01;10:86 [FREE Full text] [doi: 10.1186/1743-0003-10-86] [Medline: 24059755]

20. Korea Sports Promotion Foundation. National fitness 100 URL: http://nfa.kspo.or.kr/front/control/con0901 list.do [accessed 2020-01-28]

21. Kerr J, Anderson C, Lippman SM. Physical activity, sedentary behaviour, diet, and cancer: An update and emerging new evidence. Lancet Oncol 2017 Aug;18(8):e457-e471. [doi: 10.1016/S1470-2045(17)30411-4] [Medline: 28759385]

22. Chen X, Lu W, Zheng W, Gu K, Matthews CE, Chen Z, et al. Exercise after diagnosis of breast cancer in association with survival. Cancer Prev Res (Phila) 2011 Sep;4(9):1409-1418 [FREE Full text] [doi: 10.1158/1940-6207.CAPR-10-0355] [Medline: 21795422]

23. Friedenreich CM, Wang Q, Neilson HK, Kopciuk KA, McGregor SE, Courneya KS. Physical activity and survival after prostate cancer. Eur Urol 2016 Oct;70(4):576-585 [FRE Full text] [doi: 10.1016/j.eururo.2015.12.032] [Medline: 26774959]

24. Song M, Chan AT. The potential role of exercise and nutrition in harnessing the immune system to improve colorectal cancer survival. Gastroenterology 2018 Sep;155(3):596-600 [FREE Full text] [doi: 10.1053/j.gastro.2018.07.038] [Medline: $\underline{30076837]}$

25. Koelwyn GJ, Quail DF, Zhang X, White RM, Jones LW. Exercise-dependent regulation of the tumour microenvironment. Nat Rev Cancer 2017 Sep 25;17(10):620-632. [doi: 10.1038/nrc.2017.78] [Medline: 28943640]

26. Kirwan JP, Solomon TP, Wojta DM, Staten MA, Holloszy JO. Effects of 7 days of exercise training on insulin sensitivity and responsiveness in type 2 diabetes mellitus. Am J Physiol Endocrinol Metab 2009 Jul;297(1):E151-E156 [FREE Full text] [doi: 10.1152/ajpendo.00210.2009] [Medline: 19383872]

27. Wen C, Lin J, Yang Y, Tsai M, Tsao C, Etzel C, et al. Hepatocellular carcinoma risk prediction model for the general population: The predictive power of transaminases. J Natl Cancer Inst 2012 Oct 17;104(20):1599-1611 [FREE Full text] [doi: 10.1093/jnci/djs372] [Medline: 23073549]

28. Behrens G, Matthews CE, Moore SC, Freedman ND, McGlynn KA, Everhart JE, et al. The association between frequency of vigorous physical activity and hepatobiliary cancers in the NIH-AARP Diet and Health Study. Eur J Epidemiol 2013 Jan;28(1):55-66 [FREE Full text] [doi: 10.1007/s10654-013-9767-1] [Medline: 23354983]

29. Saran U, Humar B, Kolly P, Dufour J. Hepatocellular carcinoma and lifestyles. J Hepatol 2016 Jan;64(1):203-214. [doi: 10.1016/j.jhep.2015.08.028] [Medline: 26341826]

30. Kaibori M, Ishizaki M, Matsui K, Nakatake R, Yoshiuchi S, Kimura Y, et al. Perioperative exercise for chronic liver injury patients with hepatocellular carcinoma undergoing hepatectomy. Am J Surg 2013 Aug;206(2):202-209. [doi: 10.1016/j.amjsurg.2012.07.035] [Medline: 23374372]

31. Gill J, Baiceanu A, Clark PJ, Langford A, Latiff J, Yang P, et al. Insights into the hepatocellular carcinoma patient journey: Results of the first global quality of life survey. Future Oncol 2018 Jul;14(17):1701-1710. [doi: 10.2217/fon-2017-0715] [Medline: 29543521]

32. Ritvo P, Obadia M, Santa Mina D, Alibhai S, Sabiston C, Oh P, et al. Smartphone-enabled health coaching intervention (iMOVE) to promote long-term maintenance of physical activity in breast cancer survivors: Protocol for a feasibility pilot randomized controlled trial. JMIR Res Protoc 2017 Aug 24;6(8):e165 [FREE Full text] [doi: 10.2196/resprot.6615] [Medline: 28838886] 
33. Mendoza JA, Baker KS, Moreno MA, Whitlock K, Abbey-Lambertz M, Waite A, et al. A Fitbit and Facebook mHealth intervention for promoting physical activity among adolescent and young adult childhood cancer survivors: A pilot study. Pediatr Blood Cancer 2017 Dec;64(12):1. [doi: 10.1002/pbc.26660] [Medline: 28618158]

34. Valle CG, Deal AM, Tate DF. Preventing weight gain in African American breast cancer survivors using smart scales and activity trackers: A randomized controlled pilot study. J Cancer Surviv 2017 Feb;11(1):133-148 [FREE Full text] [doi: 10.1007/s11764-016-0571-2] [Medline: 27631874]

35. Low CA, Dey AK, Ferreira D, Kamarck T, Sun W, Bae S, et al. Estimation of symptom severity during chemotherapy from passively sensed data: Exploratory study. J Med Internet Res 2017 Dec 19;19(12):e420 [FREE Full text] [doi: 10.2196/jmir.9046] [Medline: 29258977]

36. Brown M, Murphy M, McDermott L, McAneney H, O'Sullivan JM, Jain S, et al. Exercise for advanced prostate cancer: A multicomponent, feasibility, trial protocol for men with metastatic castrate-resistant prostate cancer (EXACT). Pilot Feasibility Stud 2019;5:102 [FREE Full text] [doi: 10.1186/s40814-019-0486-6] [Medline: 31428443]

37. Egegaard T, Rohold J, Lillelund C, Persson G, Quist M. Pre-radiotherapy daily exercise training in non-small cell lung cancer: A feasibility study. Rep Pract Oncol Radiother 2019;24(4):375-382. [doi: 10.1016/j.rpor.2019.06.003] [Medline: $\underline{31289452]}$

38. Yee J, Davis GM, Hackett D, Beith JM, Wilcken N, Currow D, et al. Physical activity for symptom management in women with metastatic breast cancer: A randomized feasibility trial on physical activity and breast metastases. J Pain Symptom Manage 2019 Dec;58(6):929-939. [doi: 10.1016/j.jpainsymman.2019.07.022] [Medline: 31374368]

39. Zhang X, McClean D, Ko E, Morgan M, Schmitz K. Exercise among women with ovarian cancer: A feasibility and pre-/post-test exploratory pilot study. Oncol Nurs Forum 2017 May 01;44(3):366-374. [doi: 10.1188/17.0NF.366-374] [Medline: 28635971]

40. Xiaosheng D, Xiangren Y, Shuyuan H, Dezong G, Mengyao C, Meng D. The effects of combined exercise intervention based on Internet and social media software for postoperative patients with breast cancer: Study protocol for a randomized controlled trial. Trials 2018 Sep 06;19(1):477 [FREE Full text] [doi: 10.1186/s13063-018-2857-3] [Medline: 30189899 ]

41. Villumsen BR, Jorgensen MG, Frystyk J, Hørdam B, Borre M. Home-based 'exergaming' was safe and significantly improved 6-min walking distance in patients with prostate cancer: A single-blinded randomised controlled trial. BJU Int 2019 Oct;124(4):600. [doi: 10.1111/bju.14782] [Medline: $\underline{\text { 31012238] }}$

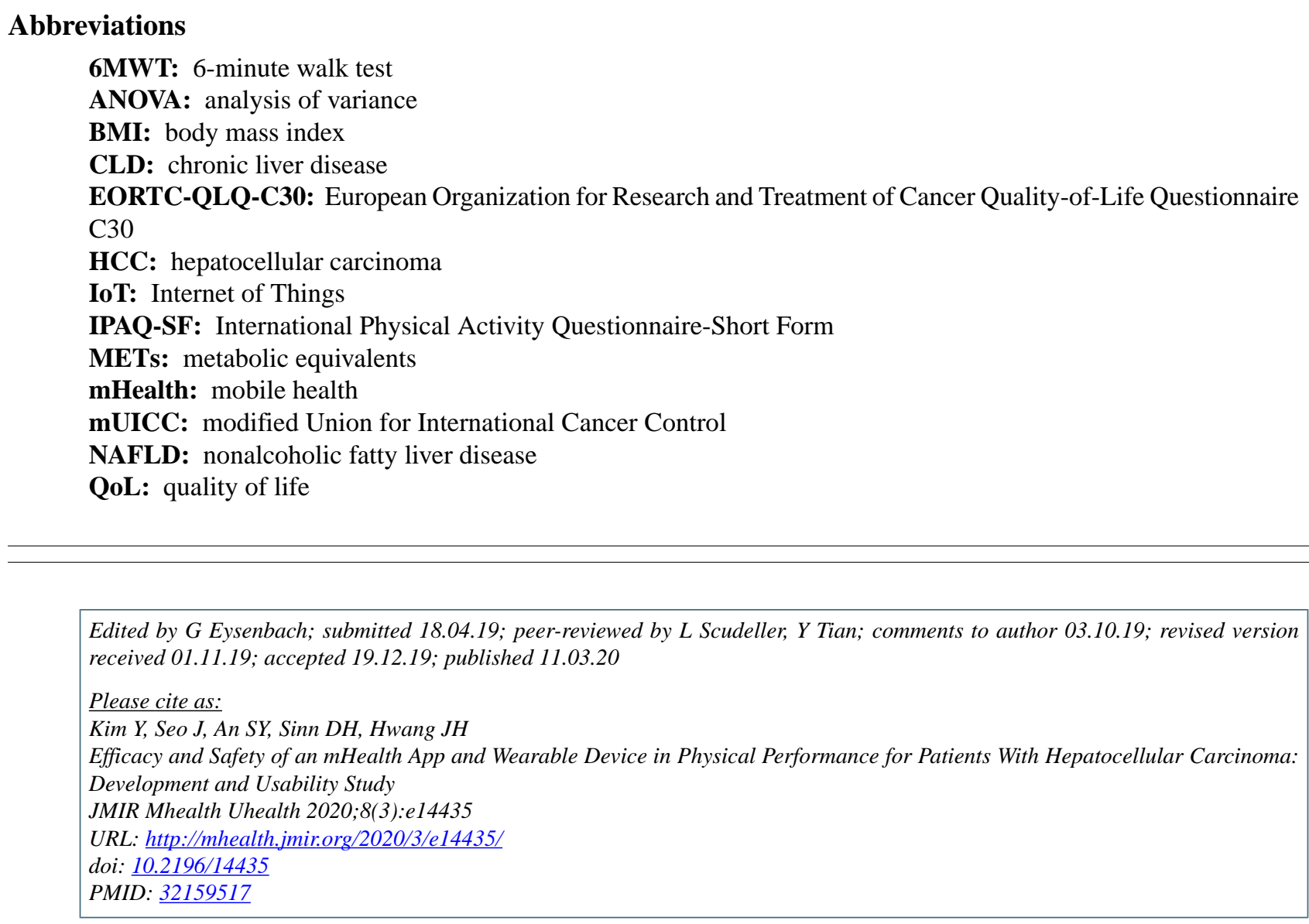


(C)Yoon Kim, Jinserk Seo, So-Yeon An, Dong Hyun Sinn, Ji Hye Hwang. Originally published in JMIR mHealth and uHealth (http://mhealth.jmir.org), 11.03.2020. This is an open-access article distributed under the terms of the Creative Commons Attribution License (https://creativecommons.org/licenses/by/4.0/), which permits unrestricted use, distribution, and reproduction in any medium, provided the original work, first published in JMIR mHealth and uHealth, is properly cited. The complete bibliographic information, a link to the original publication on http://mhealth.jmir.org/, as well as this copyright and license information must be included. 